\title{
Spin transport in germanium at room temperature
}

\author{
C. Shen ${ }^{1}{ }^{1}$ T. Trypiniotis, ${ }^{1}{ }^{1, a)}$ K. Y. Lee, ${ }^{1}$ S. N. Holmes, ${ }^{2}$ R. Mansell, ${ }^{1}$ M. Husain, ${ }^{3}$ V. Shah, ${ }^{4}$ \\ X. V. Li, ${ }^{3}$ H. Kurebayashi, ${ }^{1}$ I. Farrer, ${ }^{1}$ C. H. de Groot, ${ }^{3}$ D. R. Leadley, ${ }^{4}$ G. Bell, ${ }^{4}$ \\ E. H. C. Parker, ${ }^{4}$ T. Whall, ${ }^{4}$ D. A. Ritchie, ${ }^{1}$ and C. H. W. Barnes ${ }^{1}$ \\ ${ }^{1}$ Cavendish Laboratory, University of Cambridge, J J Thomson Avenue, CB3 OHE Cambridge, \\ United Kingdom \\ ${ }^{2}$ Cambridge Research Laboratory, Toshiba Research Europe Limited, 260 Cambridge Science Park, \\ CB4 OWE Cambridge, United Kingdom \\ ${ }^{3}$ School of Electronics and Computer Science, University of Southampton, SO17 1BJ Southampton, \\ United Kingdom \\ ${ }^{4}$ Department of Physics, University of Warwick, CV4 7AL Coventry, United Kingdom
}

(Received 27 July 2010; accepted 3 October 2010; published online 20 October 2010)

\begin{abstract}
Spin-dependent transport is investigated in a Ni/Ge/AlGaAs junction with an electrodeposited $\mathrm{Ni}$ contact. Spin-polarized electrons are excited by optical spin orientation and are subsequently used to measure the spin dependent conductance at the Ni/Ge Schottky interface. We demonstrate electron spin transport and electrical extraction from the Ge layer at room temperature. (C) 2010 American Institute of Physics. [doi:10.1063/1.3505337]
\end{abstract}

A prerequisite for the utilization of spin in a semiconductor (SC) has been the efficient injection and subsequent detection of spin polarized carriers. ${ }^{1}$ Ferromagnetic (FM) metal contacts on SCs have been studied extensively as spin detectors. ${ }^{2-5}$ Nonequilibrium spin polarization in SCs has been realized by either spin injection from a FM contact ${ }^{4,6,7}$ or optical spin orientation. ${ }^{2,3,8,9}$

The majority of spin transport investigations have concentrated on GaAs; only recently has significant progress been made toward the practical use of the spin degree of freedom in $\mathrm{Si}^{5}{ }^{5,6,10}$ Furthermore, in the other most common group IV SC, Ge, which is an attractive candidate for next generation high mobility field effect transistors, efficient spin transport has not been demonstrated to date. Several studies of charge transport across FM/Ge Schottky barrier (SB) contacts have shown strong Fermi level pinning leading to a large SB height (SBH) (Ref. 11) and subsequently a large resistance-area (RA) product $^{12-14}$ which is undesirable for spintronic applications. ${ }^{13,15}$ Attention has, therefore, turned to engineering contacts with appropriate tunnel barriers (e.g., $\mathrm{MgO}, \mathrm{Al}_{2} \mathrm{O}_{3}$ ) or in the case of SBs to treating the Ge with ion implantation in order to achieve appropriate RA values. ${ }^{13}$

The FM contacts used as spin injectors/detectors are primarily fabricated using molecular beam epitaxy (MBE) or sputtering. High quality FM/SC SB contacts can also be fabricated using electrodeposition techniques. ${ }^{16}$ As a key industrial deposition method, electrodeposition is relatively simple and cost effective, ${ }^{17}$ so establishing its use for the growth of FM contacts for spintronic devices is highly desirable.

In this paper, we demonstrate spin transport and subsequent detection of spin polarized currents in Ge at room temperature (RT) using SBs with reduced RA products formed on as-grown (untreated) Ge with electrodeposited FM contacts.

The measurements were performed on $\mathrm{Ni} / \mathrm{Ge} / n-\mathrm{Al}_{x} \mathrm{Ga}_{1-x} \mathrm{As} / \mathrm{GaAs} / p-\mathrm{Al}_{x} \mathrm{Ga}_{1-x} \mathrm{As} / p$-GaAs heterostructures $(x=0.1)$, with the $\mathrm{AlGaAs} \mathrm{n}$-i-p junction grown by MBE on a $p$-GaAs substrate (Be doped, $p=1 \times 10^{18} \mathrm{~cm}^{-3}$ )

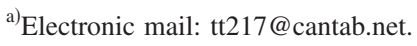

with a AuBe back Ohmic contact. ${ }^{18}$ The n-type $\mathrm{Al}_{0.1} \mathrm{Ga}_{0.9} \mathrm{As}$ layer has a Si dopant density of $2 \times 10^{18} \mathrm{~cm}^{-3}$ and was capped with As to prevent oxidation. The sample was then transferred to a second MBE chamber for the Ge growth. The As layer was first decapped at $460{ }^{\circ} \mathrm{C}$ followed by growth of $10 \mathrm{~nm}$ of $\mathrm{Ge}$ at $300{ }^{\circ} \mathrm{C}, 15 \mathrm{~nm}$ while ramping the temperature and $65 \mathrm{~nm}$ at $500{ }^{\circ} \mathrm{C}$. Native oxides on the Ge surface were removed by a 20:1 buffered HF dip for $30 \mathrm{~s}$, followed by de-ionized water wash, prior to electrodeposition of $200 \mathrm{~nm}$ of polycrystalline $\mathrm{Ni}$ in a $928 \mu \mathrm{m}$ square pad. For electrodeposition, a Ni sulfate bath and a threeelectrode potentiostat system with a Pt counter electrode and a saturated calomel reference electrode were used. ${ }^{19}$ In addition to the $\mathrm{Ni} / \mathrm{Ge}$ contact, two further contacts were made to Ge and AlGaAs using indium, as shown schematically in Fig. 1(a).

The carrier density in the Ge layer was measured on a Hall bar structure $\left(1400 \times 80 \mu \mathrm{m}^{2}\right)$ made by etching the Ge film and using Ti/Au contacts. The Hall resistance $\left(\mathrm{R}_{x y}\right)$ measurement is shown in Fig. 2 (left inset) ${ }^{20}$ giving a carrier density of $3.2 \times 10^{20} \mathrm{~cm}^{-3}$ at RT. The most probable cause of such a high carrier density is As diffusion into Ge forming n-type dopants. ${ }^{21,22}$

Figure 1(b) shows the band diagram of the sample using a self-consistent one-dimensional (1D) Schrödinger-Poisson solver $^{23}$ and a SBH of $0.5 \mathrm{eV}$ (see below). For SBs on Ge at high level of doping, field emission (FE), i.e., tunnelling, is expected to be the dominant transport mechanism. ${ }^{13,24} \mathrm{In}$ ad-

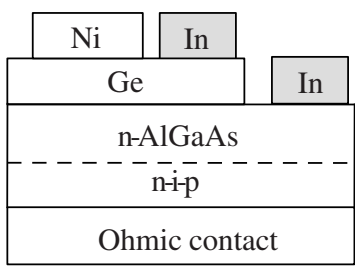

(a)

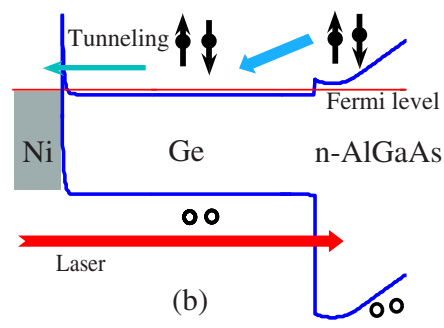

(b)
FIG. 1. (Color online) (a) A schematic cross section of the heterostructure used. (b) Simulated band diagram of the Ni/Ge/AlGaAs junction. For clarity, only the $n$-AlGaAs part of the $\mathrm{n}$-i-p junction is shown. 


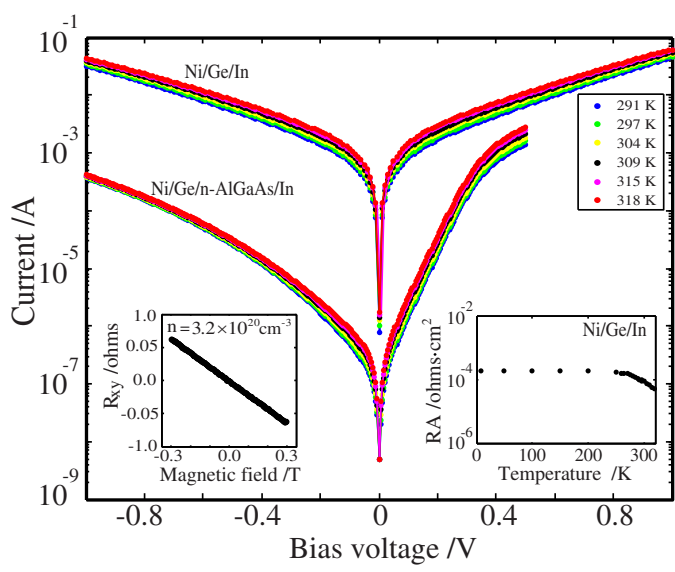

FIG. 2. (Color online) $I-V$ characteristics across the $\mathrm{Ni} / \mathrm{Ge} / \mathrm{In}$ and $\mathrm{Ni} / \mathrm{Ge} / n$-AlGaAs/In. Left inset: Hall measurement for obtaining the $\mathrm{Ge}$ carrier density. Right inset: temperature dependence of the RA product.

dition, a depletion region width of $\sim 1-2 \mathrm{~nm}$ is obtained from the simulation of Fig. 1(b) and also by a simple estimation in the depletion approximation, ${ }^{24}$ in line with FE dominant transport. $I-V$ measurements obtained from different contact combinations [Fig. 1(a)], in the temperature range $291-318 \mathrm{~K}$ are shown in Fig. 2. The symmetry in the $\mathrm{Ni} / \mathrm{Ge} / \mathrm{In} I-V$ spectra implies a back-to-back SB with similar tunnelling probabilities forming at the $\mathrm{Ni} / \mathrm{Ge}$ and $\mathrm{In} / \mathrm{Ge}$ interfaces. The temperature dependence of the back-to-back diode is limited as expected in the FE regime, similar to data on Si back-to-back diodes. ${ }^{16}$

The RA product across the $\mathrm{Ni} / \mathrm{Ge}$ interface was estimated by measuring the junction resistance for $\mathrm{Ni} / \mathrm{Ge} / \mathrm{In}$; this value would be an upper limit since it includes the resistance from the Ge channel and In/Ge interface. The exact area of current flow through the junction is difficult to determine accurately. Since the dimensions of the Ni contact are much larger than the thickness of the Ge channel, the current flow is dominated by a small distance from the edge of the contact which is approximately equal to the thickness of the $\mathrm{Ge}$ channel. ${ }^{25}$ The obtained RA product is shown in Fig. 2 (right inset) having a weak temperature dependence further confirming FE controlled transport.

Extracting the SBH in the FE regime from $I-V$ data in our back-to-back diode is problematic since it is not independent of the Richardson constant. ${ }^{24}$ In our previous work, however, a SBH of $0.52 \mathrm{eV}$ was routinely extracted from $C-V$ measurements ${ }^{24}$ on $\mathrm{Ni} / \mathrm{Ge}$ diodes with doping concentration up to $3 \times 10^{17} \mathrm{~cm}^{-3}$ and identical Ni electrodeposition conditions. ${ }^{26,27}$ Some interfacial $\mathrm{GeO}_{x}$ affecting the $\mathrm{SBH}$ might be present. ${ }^{28,29}$

The Ni/Ge/AlGaAs/In $I-V$ curve shows lower current in both bias directions. At high bias this is due to the series resistance of the AlGaAs, whereas at low bias the interface resistance of the Ge/AlGaAs is higher than that of the Ge/In interface. Although the band offset between Ge and AlGaAs is only about $0.2 \mathrm{eV}$, the larger depletion layer in $\mathrm{AlGaAs}$ prevents tunnelling in reverse bias and leads to a larger effective resistance.

Photoexcited spin polarized carriers can be generated via optical spin orientation in both the AlGaAs n-i-p and Ge layers, ${ }^{30}$ and subsequently transported through $\mathrm{Ge}$ at the $\mathrm{Ni} / \mathrm{Ge}$ interface. The polarization of the density of states of the FM gives rise to spin dependent tunnelling; the orienta-

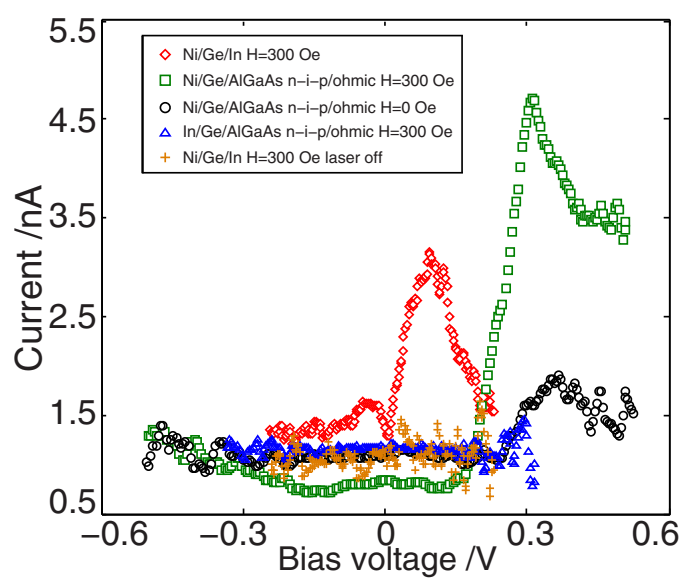

FIG. 3. (Color online) HDPC for the: Ni/Ge/In junction with applied inplane magnetic field (diamonds), $\mathrm{Ni} / \mathrm{Ge} / \mathrm{AlGaAs} \mathrm{n}$-i-p/Ohmic with applied field (squares), Ni/Ge/AlGaAs n-i-p/Ohmic without magnetic field (circles), $\mathrm{In} / \mathrm{Ge} / \mathrm{AlGaAs} \mathrm{n}-\mathrm{i}-\mathrm{p} / \mathrm{Ohmic}$ with magnetic field (triangles), and $\mathrm{Ni} / \mathrm{Ge} / \mathrm{In}$ junction with magnetic field but zero laser power (crosses).

tion of the photoexcited spin relative to the magnetization direction determines the tunnelling probability at the interface. We term this spin filtering. 2,9 All spin transport measurements were carried out at RT using laser photon energy of $1.96 \mathrm{eV}$, incident at $45^{\circ}$ to the sample plane. When required, a field of 300 Oe was applied in-plane, which practically saturates the magnetization in the Ni film as seen in magneto-optic Kerr effect measurements of the hysteresis loop.

The laser spot $(\sim 20 \mu \mathrm{m})$ was focused directly onto the Ge layer, next to the Ni pad edge. This geometry avoids transmission of light through the FM contact in order to exclude magnetic circular dichroism (MCD), the difference in the absorption of left and right circularly polarized light in a FM, which could mask any spin dependent signal. ${ }^{2,9}$ Photoexcited electrons can reach the FM contact while maintaining their spin polarization. ${ }^{31}$ The photoexcited spin polarization is reversed by altering the light helicity from left to right circular using a photoelastic modulator. The corresponding photocurrent modulation, the helicity dependent photocurrent (HDPC), was obtained using a lock-in technique. ${ }^{2}$ The HDPC is a direct measure of spin dependent transport at the $\mathrm{Ni} / \mathrm{Ge}$ interface.

The HDPC for different contact combinations is shown in Fig. 3. For the case where the laser is incident outside the $\mathrm{Ni}$ contact, directly on the $\mathrm{Ge}$, the spin filtering signal is nonzero in a small forward bias window (Fig. 3 diamonds, squares). We obtained the same behavior indirectly in previous studies after separating the MCD contribution from the data. ${ }^{2,9}$ By shining the light through the $\mathrm{Ni}$, we obtained a HDPC which increases monotonically with forward bias. This is expected for significant MCD contribution; the MCD is bias independent and the resulting HDPC signal follows the bias dependence of the photocurrent. ${ }^{9}$ For zero applied in-plane field, the corresponding reduced magnetization at remanence of the Ni film gives rise to a lower spin filtering current (Fig. 3 circles). As a reference measurement, no HDPC was observed without the Ni contact across the In/Ge/ AlGaAs/n-i-p/Ohmic junction (Fig. 3 triangles) under otherwise identical conditions. Furthermore, no signal was obtained for the $\mathrm{Ni} / \mathrm{Ge} / \mathrm{In}$ junction with the field applied and the laser off (Fig. 3 crosses). The fact that peaks in the HDPC 
signal are observed only when spin polarized carriers are injected (by the laser) and extracted through the FM contact is good evidence for spin preserving transport through the $\mathrm{Ge}$ layer and spin dependent transport across the Ge/Ni SB.

Tunnelling across the Ni/Ge $\mathrm{SB}$ is the dominant spin dependent transport process, ${ }^{2,9}$ and is maximized at the bias for which the HDPC peaks. At higher forward bias values thermionic emission is becoming more dominant and tunnelling vanishes at the flat band condition leading to a vanishing HDPC and spin dependent signal. ${ }^{2,9}$ The HDPC for the Ni/ Ge/AlGaAs n-i-p/Ohmic measurements peaks at a higher bias as compared to the Ni/Ge/In junction. The two peaks correspond to the same potential across the $\mathrm{Ni} / \mathrm{Ge}$ interface but in the former case a higher applied bias across the whole structure is required because of the potential drop across the $\mathrm{n}$-i-p and the Ge/AlGaAs junctions. At the forward bias where the spin filtering signal is maximum $(\sim 0.1 \mathrm{~V})$ we obtain an upper limit of $\mathrm{RA} \approx 6 \times 10^{-5} \Omega \mathrm{cm}^{2}$. This value is lower than that previously reported for $\mathrm{CoFeB} / \mathrm{Ge}$ contacts. ${ }^{12}$ It also compares well with values obtained in $\mathrm{Fe} / n$-Ge diodes specifically engineered for spin transport by using ion implantation on the Ge surface prior to growth. ${ }^{13}$

The absorption coefficient for Ge is $\sim 2 \times 10^{5} \mathrm{~cm}^{-1}(\lambda$ $=632.8 \mathrm{~nm}$ at RT) (Ref. 32) resulting in $83 \%$ reduction in the intensity reaching the n-i-p structure. Since the laser spot of $\sim 20 \mu \mathrm{m}$ was focused a few micrometers next to the $\mathrm{Ni}$ pad edge, there is a spatial photoexcited electron distribution in both $\mathrm{Ge}$ and AlGaAs. At the instant of photoexcitation, electrons have kinetic energies of $0.58-1.03 \mathrm{eV}$ in $\mathrm{Ge}$ and $0.05-0.36 \mathrm{eV}$ in AlGaAs obtained from direct transitions around the $\Gamma$-point. They arrive at the $\mathrm{Ni}$ film in timescales up to $\sim 20 \mathrm{ps}$, estimated by a simple ballistic transport model. The electron-lattice thermalization in Ge is $\sim 5-7 \mathrm{ps}$ between 77 and $300 \mathrm{~K},{ }^{33,34}$ and of the order of $\sim 2$ ps in GaAs at $\mathrm{RT}^{35,36} \mathrm{~A}$ significant proportion of photoexcited electrons can therefore thermalize to energies below the SBH at the $\mathrm{Ni} / \mathrm{Ge}$ interface and contribute to the spin filtering signal via tunnelling through the SB.

In summary, we have demonstrated spin dependent electron transport in Ge through a Ni/Ge/n-AlGaAs (001) structure. The Ni film, acting as the spin detector, was grown by electrodeposition, providing an experimental demonstration of the viability of using electrodeposited FM contacts for spintronic devices. We observed clear evidence of electron spin transport into and extraction from Ge at RT. The spin filtering signals are attributed to tunnelling of photoexcited electrons across the Ni/Ge SB.

This work was supported by EPSRC under Grant No. EPF0240451. In memoriam, J. A. C. Bland.

${ }^{1}$ I. Žutić, J. Fabian, and S. D. Sarma, Rev. Mod. Phys. 76, 323 (2004).

${ }^{2}$ T. Trypiniotis, D. H. Y. Tse, S. J. Steinmuller, W. S. Cho, and J. A. C. Bland, IEEE Trans. Magn. 43, 2872 (2007).

${ }^{3}$ H. Kurebayashi, T. Trypiniotis, K. Lee, S. Easton, A. Ionescu, I. Farrer, D.
Ritchie, J. A. C. Bland, and C. H. W. Barnes, Appl. Phys. Lett. 96, 022505 (2010).

${ }^{4}$ X. Lou, C. Adelmann, S. A. Crooker, E. S. Garlid, J. Zhang, S. M. Reddy, S. D. Flexner, C. J. Palmstrom, and P. A. Crowell, Nat. Phys. 3, 197 (2007).

${ }^{5}$ I. Appelbaum, B. Huang, and D. J. Monsma, Nature (London) 447, 295 (2007).

${ }^{6}$ B. T. Jonker, G. Kioseglou, A. T. Hanbicki, C. H. Li, and P. H. Thompson, Nat. Phys. 3, 542 (2007)

${ }^{7}$ A. T. Hanbicki, B. T. Jonker, G. Itskos, G. Kioseoglou, and A. Petrou, Appl. Phys. Lett. 80, 1240 (2002).

${ }^{8}$ K. Ando, M. Morikawa, T. Trypiniotis, Y. Fujikawa, C. H. W. Barnes, and E. Saitoh, Appl. Phys. Lett. 96, 082502 (2010).

${ }^{9}$ S. J. Steinmuller, C. M. Gürtler, G. Wastlbauer, and J. A. C. Bland, Phys. Rev. B 72, 045301 (2005).

${ }^{10}$ S. P. Dash, S. Sharma, R. S. Patel, M. P. de Jong, and R. Jansen, Nature (London) 462, 491 (2009).

${ }^{11}$ A. Dimoulas, P. Tsipas, A. Sotiropoulos, and E. K. Evangelou, Appl. Phys. Lett. 89, 252110 (2006).

${ }^{12}$ D. Lee, S. Raghunathan, R. J. Wilson, D. E. Nikonov, K. Saraswat, and S. X. Wang, Appl. Phys. Lett. 96, 052514 (2010).

${ }^{13}$ Y. Zhou, M. Ogawa, M. Bao, W. Han, R. K. Kawakami, and K. L. Wang, Appl. Phys. Lett. 94, 242104 (2009).

${ }^{14}$ Y. Zhou, W. Han, Y. Wang, F. Xiu, J. Zou, R. K. Kawakami, and K. L. Wang, Appl. Phys. Lett. 96, 102103 (2010).

${ }^{15}$ A. Fert and H. Jaffrès, Phys. Rev. B 64, 184420 (2001).

${ }^{16} \mathrm{M}$. Husain, X. Li, and C. H. de Groot, Solid State Commun. 149, 1565 (2009).

${ }^{17}$ L. Besra and M. Liu, Prog. Mater. Sci. 52, 1 (2007).

${ }^{18}$ Note that this heterostructure can also be used as a spin light-emitting diode which will be the focus of a future study.

${ }^{19}$ X. Li, M. Husain, M. Kiziroglou, and C. H. de Groot, Microelectron. Eng. 86, 1599 (2009).

${ }^{20}$ Note that the Hall data is corrected for the Ohmic offset, which is typical for high doping, low mobility SCs.

${ }^{21}$ V. F. Mitin, Appl. Phys. Lett. 92, 202111 (2008).

${ }^{22}$ A. Leycuras and M. G. Lee, Appl. Phys. Lett. 65, 2296 (1994).

${ }^{23}$ G. Snider, Self-consistent 1D Schrödinger-Poisson solver, http:// www.nd.edu/ gsnider/ (1997).

${ }^{24}$ S. M. Sze, Physics of Semiconductor Devices, 2nd ed. (Wiley, New York, 1981).

${ }^{25}$ H. Murrmann and D. Widmann, IEEE Trans. Electron Devices 16, 1022 (1969).

${ }^{26}$ M. K. Husain, X. Li, and C. H. de Groot, IEEE Trans. Electron Devices 56, 499 (2009).

${ }^{27} \mathrm{M}$. Husain, X. V. Li, and C. H. de Groot, IEEE Electron Device Lett. 30, 966 (2009).

${ }^{28}$ T. Nishimura, K. Kita, and A. Toriumi, Appl. Phys. Express 1, 051406 (2008).

${ }^{29}$ M. Kobayashi, A. Kinoshita, K. Saraswat, H.-S. Philip Wong, and Y. Nishi, J. Appl. Phys. 105, 023702 (2009).

${ }^{30}$ F. Meier and B. P. Zakharchenya, Optical Orientation (North-Holland, Amsterdam, 1984).

${ }^{31}$ S. A. Crooker, E. S. Garlid, A. N. Chantis, D. L. Smith, K. S. M. Reddy, Q. O. Hu, T. Kondo, C. J. Palmstrom, and P. A. Crowell, Phys. Rev. B 80, 041305 (2009).

${ }^{32}$ H. P. Philipp and E. A. Taft, Phys. Rev. 113, 1002 (1959).

${ }^{33}$ A. Othonos, H. M. van Driel, J. F. Young, and P. J. Kelly, Phys. Rev. B 43, 6682 (1991).

${ }^{34}$ H. Roskos, B. Rieck, A. Seilmeier, and W. Kaiser, Appl. Phys. Lett. 53, 2406 (1988).

${ }^{35}$ N. Del Fatti, P. Langot, R. Tommasi, and F. Vallée, Phys. Rev. B 59, 4576 (1999).

${ }^{36}$ P. Langot, N. Del Fatti, D. Christofilos, R. Tommasi, and F. Vallée, Phys. Rev. B 54, 14487 (1996). 